\title{
Transversity and SIDIS at Jefferson Lab
}

\author{
Jen-Chieh Peng*, Jian-Ping Chen ${ }^{\dagger}$, Xiaodong Jiang** and Lingyan Zhu* \\ ${ }^{*}$ University of Illinois at Urbana-Champaign, Urbana, Illinois, 61801, U.S.A. \\ $\dagger$ Jefferson Lab, Newport News, Virginia, 23606, U.S.A. \\ ${ }^{* *}$ Rutgers University, Piscataway, New Jersey, 08855, U.S.A.
}

\section{INTRODUCTION}

Remarkable progress in the study of spin-dependent structure functions has been made since the discovery of the "proton spin puzzle" in the late 1980's. Very active spinphysics programs have been pursued at many facilities including SLAC, CERN, HERA, JLab, and RHIC. In recent years, new experimental tools such as semi-inclusive polarized DIS, polarized proton-proton collision, and deeply exclusive reactions have been employed to address various aspects of the proton spin. In this paper, we present the plans at the Jefferson Lab to use the semi-inclusive DIS (SIDIS) reactions to measure various unpolarized and polarized nucleon parton distribution and fragmentation functions. In addition, the prospect of probing the flavor structure of the kaon fragmentation function is discussed.

\section{TRANSVERSITY}

The nucleon unpolarized and polarized quark distributions, $q\left(x, Q^{2}\right)$ and $\Delta q\left(x, Q^{2}\right)$, are now rather well known. In contrast, a third quark distribution, called transversity, is the remaining twist-2 distribution which is practically unmeasured. This helicityflip quark distribution, $\delta q\left(x, Q^{2}\right)$, can be described in quark-parton model as the net transverse polarization of quarks in a transversely polarized nucleon [1]. The transversity distributions have many interesting theoretical aspects, related to general properties of QCD as well as to the structure of the nucleon:

- In the non-relativistic quark model, where boosts and rotations commute, the transversity distributions are identical to the longitudinally polarized distributions. The transversity distributions provide a detailed measure of the relativistic nature of the quarks inside the nucleon.

- The transversity distribution is expected to have a valence like behavior. Since the transversity of gluons in a nucleon does not exist, the transversity distribution is expected to follow a simple evolution as a flavor non-singlet quantity [2].

- The transversity obeys some important inequalities. The first, $\left|h_{1}^{q}\right| \leq f_{1}^{q}$, follows from its interpretation as a difference of probabilities. The second (Soffer's bound) 
has its origins in the positivity of helicity amplitudes [3]: $\left|h_{1}^{q}\right| \leq \frac{1}{2}\left(f_{1}^{q}+g_{1}^{q}\right)$.

- The lowest moment of $h_{1}^{q}$ measures a simple local operator analogous to the axial charge, known as the "tensor charge".

Due to the chiral-odd nature of the transversity distribution, it can not be measured in inclusive DIS experiments. In order to measure $\delta q\left(x, Q^{2}\right)$, an additional chiral-odd object is required. For example, the double spin asymmetry, $A_{T T}$, for Drell-Yan cross section in transversely polarized $p p$ collision, is sensitive to transversity since $A_{T T} \sim$ $\sum_{i} e_{i}^{2} \delta q_{i}\left(x_{1}\right) \delta \bar{q}_{i}\left(x_{2}\right)$. Such a measurement could be carried out at RHIC [4], although the anticipated effect is small, on the order of $1-2 \%$.

Several other methods for measuring transversity have been proposed for semiinclusive DIS. In particular, Collins suggested [5] that a novel chiral-odd fragmentation function (Collins function) in conjunction with the chiral-odd transversity distribution would lead to a single-spin azimuthal asymmetry in semi-inclusive pion production. Results on target single spin azimuthal asymmetries in SIDIS reactions from SMC [6] and HERMES [7] offered the first glimpse of possible effects caused by the transversity distribution.

The semi-inclusive DIS is a powerful tool for probing various parton distribution and fragmentation functions. For producing a spin-zero meson, the SIDIS differential cross section at the leading order is $[8,9]$ :

$$
\begin{aligned}
d^{6} \sigma^{L O}= & \frac{d \sigma^{\ell+N \rightarrow \ell^{\prime}+h+X}}{d x d y d \phi^{\ell} d z d^{2} P_{h \perp}}=\frac{4 \pi \alpha^{2} s x}{Q^{4}} \times \\
& \left\{\left[1+(1-y)^{2}\right] \sum_{q, \bar{q}} e_{q}^{2} f_{1}^{q}(x) D_{1}^{q}\left(z, P_{h \perp}^{2}\right)\right. \\
& +(1-y) \frac{P_{h \perp}^{2}}{4 z^{2} M_{N} M_{h}} \cos \left(2 \phi_{h}^{\ell}\right) \sum_{q, \bar{q}} e_{q}^{2} h_{1}^{\perp(1) q}(x) H_{1}^{\perp q}\left(z, P_{h \perp}^{2}\right) \\
& -\left|S_{L}\right|(1-y) \frac{P_{h \perp}^{2}}{4 z^{2} M_{N} M_{h}} \sin \left(2 \phi_{h}^{\ell}\right) \sum_{q, \bar{q}} e_{q}^{2} h_{1 L}^{\perp(1) q}(x) H_{1}^{\perp q}\left(z, P_{h \perp}^{2}\right) \\
& +\left|S_{T}\right|(1-y) \frac{P_{h \perp}}{z M_{h}} \sin \left(\phi_{h}^{\ell}+\phi_{S}^{\ell}\right) \sum_{q, \bar{q}} e_{q}^{2} h_{1}^{q}(x) H_{1}^{\perp q}\left(z, P_{h \perp}^{2}\right) \\
& +\left|S_{T}\right|\left(1-y+\frac{1}{2} y^{2}\right) \frac{P_{h \perp}}{z M_{N}} \sin \left(\phi_{h}^{\ell}-\phi_{S}^{\ell}\right) \sum_{q, \bar{q}} e_{q}^{2} f_{1 T}^{\perp(1) q}(x) D_{1}^{q}\left(z, P_{h \perp}^{2}\right) \\
& +\left|S_{T}\right|(1-y) \frac{P_{h \perp}^{3}}{6 z^{3} M_{N}^{2} M_{h}} \sin \left(3 \phi_{h}^{\ell}-\phi_{S}^{\ell}\right) \sum_{q, \bar{q}} e_{q}^{2} h_{1 T}^{\perp(2) q}(x) H_{1}^{\perp q}\left(z, P_{h \perp}^{2}\right) \\
& +\lambda_{e}\left|S_{L}\right| y\left(1-\frac{1}{2} y\right) \sum_{q, \bar{q}} e_{q}^{2} g_{1}^{q}(x) D_{1}^{q}\left(z, P_{h \perp}^{2}\right) \\
& \left.+\lambda_{e}\left|S_{T}\right| y\left(1-\frac{1}{2} y\right) \frac{P_{h \perp}}{z M_{N}} \cos \left(\phi_{h}^{\ell}-\phi_{S}^{\ell}\right) \sum_{q, \bar{q}} e_{q}^{2} g_{1 T}^{(1) q}(x) D_{1}^{q}\left(z, P_{h \perp}^{2}\right)\right\},
\end{aligned}
$$


where $x, y, z, Q^{2}$ are the familiar kinematic variables. $\left|S_{L}\right|$ and $\left|S_{T}\right|$ are the longitudinal and transverse target spin components with respect to the virtual photon direction, and $\lambda_{e}$ is the electron helicity. Azimuthal angles are defined relative to the lepton plane, e.g. $\phi_{h}^{\ell}=\phi_{h}-\phi^{\ell}$ is the angle between the hadron plane $\left(\vec{P}_{h} \wedge \vec{q}\right)$ and the lepton plane. $\phi_{S}^{\ell}=\phi_{S}-\phi^{\ell}$ is the angle between the $\vec{S} \wedge \vec{q}$ plane and the lepton plane. $P_{h \perp}$ is the component of the hadron's momentum perpendicular to the virtual photon direction.

In Eq. $1, f_{1}^{q}(x), g_{1}^{q}(x)$ and $h_{1}^{q}(x)$ are the helicity-averaged, helicity-dependent, and transversity distribution functions, respectively. These three functions survive the integration of intrinsic quark transverse momentum $p_{T}$ in the initial nucleon. The other $p_{T}$-dependent distributions are $f_{1 T}^{\perp q}, g_{1 T}^{\perp q}, h_{1}^{\perp q}, h_{1 L}^{\perp q}$ and $h_{1 T}^{\perp q}$. They only survive the $p_{T}$ integration after weighting with $\left(p_{T}^{2} / 2 M_{N}^{2}\right)^{n}$. Such weighting is indicated by a superscript (n) in Eq. 1. $D_{1}^{q}$ and $H_{1}^{\perp q}$ are the regular fragmentation function and the chiral-odd, T-odd Collins fragmentation function, respectively.

Eq. 1 shows that SIDIS can be used to extract all eight of the twist-2 structure functions with a judicial selection of beam helicity $\left(\lambda_{e}\right)$, target polarization $\left(S_{L}, S_{T}\right)$, and the detection of azimuthal angular dependences $\left(\phi_{h}^{l}\right.$ and $\left.\phi_{s}^{l}\right)$.

The HERMES collaboration recently reported [7] observation of single-spin azimuthal asymmetry for charged and neutral hadron electroproduction. Using unpolarized positron beam on longitudinally polarized hydrogen and deuterium targets, the cross section was found to have a $\sin \phi_{h}^{l}$ dependence. Although a longitudinally polarized target was used in the HERMES experiment, $S_{T}$ is nonzero with a mean value of $\approx 0.15$. Eq. 1 shows that a $\sin \phi_{h}^{l}$ dependence can arise from the transversity plus collins fragmentation function (the $\sin \left(\phi_{h}^{l}+\phi_{s}^{l}\right)$ term), as well as from $f_{1 T}^{\perp}$, the chiral-odd T-odd Sivers distribution function [10] (the $\sin \left(\phi_{h}^{l}-\phi_{s}^{l}\right)$ term). The Collins fragmentation function represents a correlation between the quark's transverse spin and the transverse momentum of the leading hadron formed in the fragmentation process. The Sivers distribution function reflects the correlation between the quark's transverse spin and its transverse momentum within the nucleon. For a longitudinally polarized target $\left(\phi_{s}^{l}=0\right)$ the Collins and the Sivers mechanisms can not be distinguished.

If the azimuthal asymmetry observed by HERMES is indeed caused by the nucleon transversity, a much larger asymmetry is expected for a transversely polarized target. The HERMES [11] and COMPASS [12] collaborations have collected polarized SIDIS using transversely polarized hydrogen and LiD targets, respectively. A simultaneous fit to $\sin \left(\phi_{h}^{l}+\phi_{s}^{l}\right)$ and $\sin \left(\phi_{h}^{l}-\phi_{s}^{l}\right)$ dependences was applied to the HERMES data to extract the "Collins moments" and the "Sivers moments", respectively. Nonzero Collins moments were clearly observed in the HERMES data with positive azimuthal asymmetry for $\pi^{+}$and negative azimuthal asymmetry for $\pi^{-}$. The unexpectedly large magnitude for the azimuthal asymmetry of $\pi^{-}$relative to $\pi^{+}$seems to suggest that the disfavored Collins function is of comparable magnitude, but opposite sign, to the favored Collins function. Furthermore, the observation of the $\sin \left(\phi_{h}^{l}-\phi_{s}^{l}\right)$ moments shows that the Sivers function is nonzero and indeed contribute to the azimuthal asymmetry in SIDIS. Preliminary result [12] from the COMPASS 2002 data shows that the $\sin \left(\phi_{h}^{l}+\phi_{s}^{l}\right)$ azimuthal asymmetry is consistent with zero for transversely polarized LiD target. A factor of 4 increase in statistics is expected for the COMPASS 2002-2004 data. 


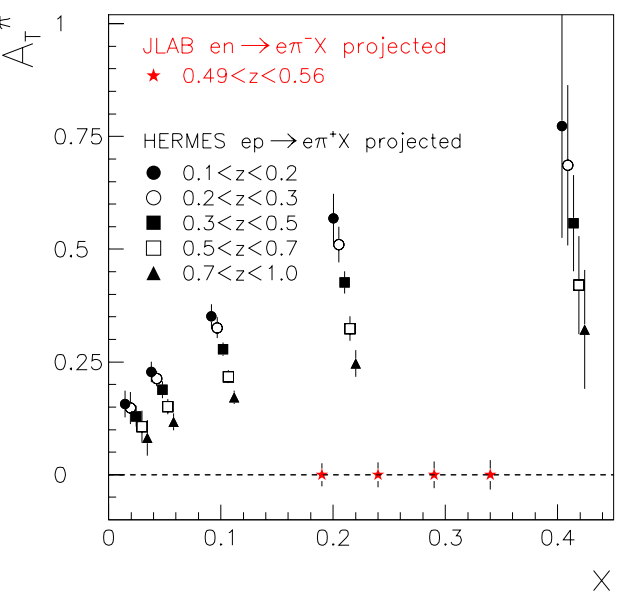

FIGURE 1. Expected statistical precision of this experiment in comparison with HERMES run-II projected precision on a proton target [14].

In order to confront theoretical predictions on the transversity distribution, precise SIDIS data on both proton and neutron covering a wide range of kinematics is required. A recently approved JLab proposal [13] plans to measure the single spin asymmetry of the $\vec{n}\left(e, e^{\prime} \pi^{-}\right) X$ reaction on a transversely polarized ${ }^{3} \mathrm{He}$ target. The goal of this experiment is to provide the first measurement on the neutron transversity, complementary to the ongoing HERMES and COMPASS measurements on the proton and deuteron. This experiment focuses on the valence quark region, $x=0.19 \sim 0.34$, at $Q^{2}=1.77 \sim 2.73$ $\mathrm{GeV}^{2}$. This kinematics is comparable to the HERMES measurement. The variation of single spin asymmetry relative to $x$ and $z$ will isolate effect of parton distribution from that of fragmentation. Data from this experiment, when combined with data from HERMES and COMPASS, will provide powerful constraints on the transversity distributions on both $u$-quark and $d$-quark in the valence region.

The experiment will use $6 \mathrm{GeV}$ electron beam with the Hall A left-side high resolution spectrometer $\left(\mathrm{HRS}_{L}\right)$ situated at $16^{\circ}$ as the hadron arm, and the BigBite spectrometer located at $30^{\circ}$ beam-right as the electron arm. A Rb spin-exchange ${ }^{3} \mathrm{He}$ target of $50 \mathrm{mg} / \mathrm{cm}^{2}$ and $\sim 42 \%$ transverse polarization with tunable direction will be used. By rotating the target polarization direction, the coverage in $\phi_{s}^{l}$ is increased, hence facilitating the separation of the Collins and the Sivers effects. Figure 1 shows the expected statistical precision of this experiment for $\vec{n}\left(e, e^{\prime} \pi^{-}\right) X$ single spin asymmetry together with the projected precision[14] of HERMES run-II of the $\vec{p}\left(e, e^{\prime} \pi^{+}\right) X$ reaction.

\section{The $h_{1}^{\perp}$ quark distributions}

In addition to the Svers function, the other T-odd quark distribution is the $h_{1}^{\perp}(x)$ function. $h_{1}^{\perp}$ is interpreted as the distribution of a transversely polarized quark with nonzero transverse momentum inside an unpolarized nucleon. Eq. 1 shows that the $h_{1}^{\perp}(x)$ 
can be extracted by isolating the $\cos \left(2 \phi_{h}^{l}\right)$ azimuthal dependence. It would be of great interest if existing and future SIDIS experiments can shed some light on the T-odd $h_{1}^{\perp}(x)$ quark distributions.

$h_{1}^{\perp}$ can also lead to a $\cos 2 \phi$ dependence in unpolarized Drell-Yan process [15]. The azimuthal angle in this case is the angle between $l^{-}$and the plane formed by the beam and the virtual photon. As a time-odd quantity, $h_{1}^{\perp}$ in the Drell-Yan process is predicted [16] to have an opposite sign relative to the $h_{1}^{\perp}$ in the SIDIS process. However, this prediction remains to be confirmed by experiments. A large $\cos 2 \phi$ dependence was observed in pion-induced Drell-Yan process, and a first attempt to extract $h_{1}^{\perp}$ information from these data has been made [15]. The $\cos 2 \phi$ dependence has never been extracted for the proton-induced Drell-Yan data. The recent FNAL E866 experiment has collected $\sim 2.5 \times 10^{5} p+p$ and $p+d$ Drell-Yan events using $800 \mathrm{GeV} / \mathrm{c}$ proton beams. These data are being analysed to determine the $\cos 2 \phi$ dependence. A comparison of proton Drell-Yan data with the pion Drell-Yan data could provide additional light on the role of $h_{1}^{\perp}$ on the $\cos 2 \phi$ dependence in the Drell-Yan process.

\section{Other SIDIS initiaves at JLab}

A new JLab Hall-C approved proposal [17] plans to measure spin asymmetries in $\vec{e} \vec{p} \rightarrow e^{\prime} h X$ and $\vec{e} \vec{d} \rightarrow e^{\prime} h X$ SIDIS reactions. Taking advantage of the high flux electron beam and a large-acceptance BETA spectrometer for electron detection, this experiment aims at a high statistics measurement of $\Delta u, \Delta d, \Delta \bar{u}, \Delta \bar{d}$ over the $0.1<x<0.45$ range. This experiment could address the important unresolved issue on the flavor structure of polarized sea-quark distributions. Recent polarized SIDIS results from HERMES have already provided a first measurement of the $\Delta \bar{u}$ and $\Delta \bar{d}$ [18].

Another new JLab proposal [19] plans to measure unpolarized $\left(e, e^{\prime} \pi^{ \pm}\right)$and $\left(e, e^{\prime} K^{ \pm}\right)$ reactions. The pion SIDIS measurement aims at a determination of $\bar{d}-\bar{u}$ with better statistical accuracy than the existing HERMES data [20]. The kaon SIDIS measurement is motivated by a search for the $\bar{u}, \bar{d}$ asymmetry in the kaon fragmentation function. The interest for such a study is discussed in the next Section.

\section{FLAVOR ASYMMETRY OF FRAGMENTATION FUNCTIONS}

The $\bar{d} / \bar{u}$ asymmetry in the proton is now well established [21]. It is of interest to examine the flavor structure of sea quark distributions in other hadrons. Alberg et al. [22] have considered the sea-quark distributions in $\Sigma^{+}$. The meson-cloud model implies a $\bar{d} / \bar{u}$ asymmetry in the $\Sigma^{+}$even larger than that of the proton. The opposite effect, however, is expected from SU(3) symmetry. A Drell-Yan experiment using a $\Sigma^{+}$hyperon beam has been considered [22] for such study, but appears quite challenging.

An alternative area of investigation is in the meson sector. A flavor asymmetry in the $\pi^{+}$sea would show up as $u_{\text {sea }} \neq d_{\text {sea }}$, which is equivalent to $\bar{u} \neq d$. However, the chargeconjugation symmetry and isospin symmetry dictate that $\bar{u}=d$ in the $\pi^{+}$. Therefore, the sea quark distributions in charged pions are $u / d$ symmetric. This is readily understood 
in the Pauli-blocking picture. The presence of the $u$ and $\bar{d}$ valence quarks in $\pi^{+}$would equally block the $u \bar{u}$ and the $\bar{d} d$ sea.

The situation is quite different for charged kaons. One anticipates an $\bar{d} / \bar{u}$ asymmetry based on the Pauli-blocking picture. The presence of $u$ quark in $K^{+}$would inhibit the $\bar{u}$ sea relative to the $\bar{d}$ sea in $K^{+}$. Therefore, one expects $\bar{d}>\bar{u}$ in $K^{+}$, analogous to the situation for proton. Unfortunately, the putative $\bar{u} / \bar{d}$ flavor asymmetry in $K^{+}$is not accessible in DIS experiments.

A promising avenue for probing the possible $\bar{u} / \bar{d}$ asymmetry in $K^{+}$is the measurement of kaon fragmentation functions. The sea-quark flavor asymmetry would imply that $\bar{u}$ and $\bar{d}$ would have unequal probability to hadronize into a $K^{+}$, namely, $D_{\bar{u}}^{K^{+}} \neq D_{\bar{d}}^{K^{+}}$. A possible evidence for such asymmetry might already exist. Kaon fragmentation functions have been extracted by Kniehl et al. [23] who fitted the hadron production data from $e^{+} e^{-}$annihilation. They obtain the following surprising result:

$$
\int_{0.05}^{1} d x \times D_{u, s}^{K^{ \pm}}\left(x, Q_{0}^{2}\right)=0.19, \quad \int_{0.05}^{1} d x \times D_{u, s}^{K^{ \pm}}\left(x, Q_{0}^{2}\right)=0.25
$$

This result shows that the $d$ quark, which is a sea quark for $K^{ \pm}$, has a higher probability to fragment into $K^{ \pm}$than for the valence $u$ and $s$ quarks. This surprising result might reflects a large $\bar{d} / \bar{u}$ flavor asymmmtry in the kaon sea. Clearly, additional data on kaon fragmentation function would be of great interest. The JLab proposal [19] plans to measure kaon SIDIS to examine possible flavor asymmetry of the kaon fragmentation functions.

\section{REFERENCES}

1. Barone, V., Drago, A., and Ratcliffe, P., Phys. Rep., 359, 1 (2002).

2. Bourrely, C., Soffer, J., and Teryaev, O. V., Phys. Lett., B420, 375 (1998).

3. Soffer, J., Phys. Rev. Lett., 74, 1292 (1995).

4. Bunce, G., et al., Ann. Rev. Nucl. Part. Sci., 50, 525 (2000).

5. Collins, J., Nucl. Phys., B396, 161 (1993).

6. Bravar, A., et al., Nucl. Phys. Proc. Suppl., 79, 520 (1999).

7. Airapetian, A., et al., Phys. Lett., B562, 182 (2003).

8. Mulders, P., and Tangerman, R. D., Nucl. Phys., B461, 197 (1996).

9. Boer, D., and Mulders, P., Phys. Rev., D57, 5780 (1998).

10. Sivers, D. W., Phys. Rev., D41, 83 (1990).

11. Airapetian, A., et al., hep-ex/0408013 (2004).

12. Bressan, A., Spin2004 Proceedings (2004).

13. Chen, J.-P., Jiang, X., Peng, J., et al., JLab proposal PR-03-014 (2003).

14. Korotkov, V., Nowak, W.-D., and Oganessian, K., Eur. Phys. J., C18, 639 (2001).

15. Boer, D., Phys. Rev., D60, 014012 (1999).

16. Boer, D., Brodsky, S., and Hwang, D., Phys. Rev., D67, 054003 (2003).

17. Jiang, X., et al., JLab proposal PR-04-113 (2004).

18. Airapetian, A., et al., Phys. Rev. Letts., 92, 012005 (2004).

19. Zhu, L., et al., JLab proposal PR-04-114 (2004).

20. Ackerstaff, K., et al., Phys. Rev. Letts., 81, 5519 (1998).

21. Garvey, G. T., and Peng, J. C., Prog. Part. Nucl. Phys. Phys., 47, 203 (2001).

22. Alberg, M., et al., Phys. Lett., B389, 367 (2003).

23. Kniehl, B., Kramer, G., and Potter, B., Nucl. Phys., B582, 514 (2000). 